\title{
Sedación en paciente con superobesidad: reporte de caso
}

\section{Sedation in a patient with super obesity: Case report}

\author{
Rosalba Olvera-Martínez, Nancy G. Loredo-García* y Beatriz Mutis-Ospino
}

Servicio de Anestesiología, Hospital General Dr. Manuel Gea González, Ciudad de México, México

\section{Resumen}

La obesidad se asocia con mayor morbimortalidad. La sedación en procedimientos para estos pacientes puede implicar desafíos importantes. La sedación consciente es la causa de muchos eventos no planificados potencialmente graves durante la endoscopia. Se presenta el reporte de un caso de sedación con propanidido en un paciente con superobesidad para un procedimiento endoscópico. El propanidido ha demostrado su seguridad y eficacia en procedimientos cortos en personas obesas.

Palabras clave: Superobesidad. Obesidad. Sedación. Endoscopia. Bariatría. Propanidido.

\begin{abstract}
Obesity is associated with increased morbidity and mortality. Sedation of the procedure for these patients can pose significant challenges. Conscious sedation is the cause of many potentially serious unplanned events during endoscopy. A case report of sedation with propanidid in a patient with super obesity for endoscopic procedure is presented. Propanidid has demonstrated its safety and efficacy in short procedures in obese people.
\end{abstract}

Key words: Super-obesity. Obesity. Sedation. Endoscopy. Bariatrics. Propanidid.

\section{Introducción}

La Organización Mundial de la Salud clasifica la obesidad como grado I (índice de masa corporal [IMC] 30-34 kg/m²), grado II (IMC $\left.35-39 \mathrm{~kg} / \mathrm{m}^{2}\right)$ o grado III $\left(\mathrm{IMC}>40 \mathrm{~kg} / \mathrm{m}^{2}\right)$, superobesidad $\left(\mathrm{IMC}>50 \mathrm{~kg} / \mathrm{m}^{2}\right)$ y super-superobesidad $\left(\mathrm{IMC}>60 \mathrm{~kg} / \mathrm{m}^{2}\right)^{1,2}$. La obesidad está asociada con una mayor morbimortalidad. Los tratamientos de primera línea incluyen modificaciones en la dieta y actividad física; la cirugía bariátrica es otra opción. Muchos pacientes se someten a una endoscopia superior ambulatoria, y la sedación para el procedimiento en estos pacientes puede implicar desafíos importantes ${ }^{3}$. Es posible realizarla con éxito bajo sedación consciente (el paciente responde a órdenes verbales o estimulación táctil) y mejora la tolerancia de la endoscopia, pero también es causa de muchos eventos no planificados potencialmente graves, de los cuales el más común es la hipoxemia, que ocurre en un $10-70 \%$ de los casos, la mayoría transitorios y con respuesta al oxígeno suplementario; sin embargo, puede llevar a isquemia cardiaca con

\section{Correspondencia:}

*Nancy G. Loredo-García

Avda. Calzada de Tlalpan 4800

Sección XVI

C.P. 14080, Ciudad de México, México

E-mail: nancy.loredo.garcia@ hotmail.com
Fecha de recepción: 08-10-2020

Cir Cir. 2021;89(S1):49-53

Fecha de aceptación: 10-03-2021

DOI: $10.24875 / C I R U .20001100$
Contents available at PubMed www.cirugiaycirujanos.com

0009-7411/@ 2020 Academia Mexicana de Cirugía. Publicado por Permanyer. Este es un artículo open access bajo la licencia CC BY-NC-ND (http://creativecommons.org/licenses/by-nc-nd/4.0/). 
depresión del segmento ST en personas con o sin enfermedad cardiaca preexistente ${ }^{4,5}$. Un IMC más alto se asocia con mayor riesgo en un procedimiento bajo sedación, así como el síndrome de apnea obstructiva del sueño (SAOS), la hipertensión pulmonar y la enfermedad pulmonar restrictiva. El manejo de la vía aérea en los pacientes obesos puede resultar más difícil debido a la rápida desaturación de oxígeno, los desafíos con la ventilación y la intubación ${ }^{3}$. En un análisis de regresión logística multivariable, el IMC (odds ratio [OR]: 2.0; intervalo de confianza del 95\% [IC95\%]: 1.3-3.1; $p<0.001$ y la edad (OR: 1.1; IC95\%: 1.0-1.1; $p=0.02$ ) fueron predictores independientes de cualquier maniobra de la vía aérea y de complicaciones relacionadas con la sedación ${ }^{6,7}$. En los obesos, el propofol acentúa el colapso de las vías respiratorias, lo que puede aumentar el riesgo de eventos adversos cardiopulmonares ${ }^{6}$. Los sujetos de alto riesgo con SAOS tienen más probabilidades de presentar desaturación y episodios hipercápnicos cuando se someten a sedación para endoscopia ${ }^{8}$. La incidencia de SAOS en los pacientes con obesidad mórbida es del $64 \%$, y aumenta con la gravedad de la obesidad ${ }^{9}$. Recibir tratamiento para el SAOS conlleva una menor incidencia de complicaciones anestésicas perioperatorias $^{9}$. Ante el panorama que enfrentamos, es necesario valorar diversos medicamentos para llevar a cabo los procedimientos anestésicos.

\section{Caso clínico}

Varón de 46 años. Presión arterial 128/71 mmHg, frecuencia cardiaca 113 latidos por minuto (I.p.m.), SpO2 88-91\%, peso $242.2 \mathrm{~kg}$, talla $165 \mathrm{~cm}$, IMC $89 \mathrm{~kg} / \mathrm{m}^{2}$. Acude a consulta para valoración preanestésica en clínica de bariatría. Antecedentes personales patológicos: super-superobesidad (grado IV), hipertensión arterial de 10 años de evolución en tratamiento con lisinopril $10 \mathrm{mg} / 24 \mathrm{~h}$ y clortalidona $50 \mathrm{mg} / 24 \mathrm{~h}$, en adecuado control. Resistencia a la insulina de 9 años de evolución en tratamiento con metformina $850 \mathrm{mg} / 8 \mathrm{~h}$. Hiperuricemia de 25 años de evolución, última crisis gotosa hace 6 años, tratada con alopurinol $300 \mathrm{mg} / 24 \mathrm{~h}$, que suspendió hace 3 años por indicación médica. SAOS de 9 años de evolución con indicación de presión positiva continua en las vías respiratorias (CPAP), que nunca usó por decisión propia. Neuralgia del nervio ciático de 13 años de evolución en tratamiento con ketorolaco sublingual ocasional. Antecedentes personales no patológicos: alergias, transfusiones y traumatismos negados.
Tabaquismo positivo de 30 años de evolución, índice tabáquico 10.7. Alcoholismo positivo de 30 años de evolución, consumo moderado de manera semanal. Sedentario. Antecedente quirúrgico de fístula anal en 1992 bajo bloqueo neuroaxial, sin complicaciones. Exploración física: Glasgow 15, orientado en espacio y tiempo. Ruidos cardiacos rítmicos sin soplos ni agregados, campos pulmonares bien ventilados, no estertores ni sibilancias. Abdomen con abundante panículo adiposo. Insuficiencia venosa superficial y profunda de miembros pélvicos; en el tobillo izquierdo presenta una úlcera posterior de unos $5-6 \mathrm{~cm}$ de diámetro, sin datos de infección. Apertura bucal mayor de $3 \mathrm{~cm}$, Mallampati I, Bellhouse II, Patil-Aldreti I, distancia esternomentoniana $16 \mathrm{~cm}$, circunferencia de cuello $53 \mathrm{~cm}$. Capacidad funcional < 4 met, Stop Bang 7 puntos, Obese 2 puntos, Ariscat bajo, Caprini alto, índice de Lee I. Los resultados de laboratorios solicitados se muestran en la tabla 1. Estudios de gabinete: electrocardiograma con ritmo sinusal, frecuencia cardiaca de 90 I.p.m., sin trastornos del ritmo ni de la conducción, sin datos de isquemia. Radiografía de tórax normal. No cuenta con ecocardiograma transtorácico debido a la imposibilidad de realizarlo por su IMC. Debido a la baja reserva pulmonar y las condiciones del paciente, no es posible realizar ecocardiograma transesofágico bajo sedación. Paciente con diagnóstico de super-superobesidad candidato para bypass gástrico, pero por su elevado IMC no es posible realizar la cirugía laparoscópica por imposibilidad de introducir los trocares. Se decide colocarle un balón intragástrico endoscópico para ayudar a la reducción de peso y así posteriormente realizar estudios para completar el protocolo y llevar a cabo el procedimiento quirúrgico. Se inicia CPAP, $8 \mathrm{cmH}_{2} \mathrm{O}$, progresión de rampa cada 30 minutos, por las noches durante 8 semanas, alopurinol $150 \mathrm{mg} / 24 \mathrm{~h}$ y dieta de $2100 \mathrm{kcal}$. Tras 2 meses de preparación, se decide colocarle el balón intragástrico. El día del procedimiento, el paciente presenta peso $240 \mathrm{~kg}$, talla $165 \mathrm{~cm}$, IMC $88.2 \mathrm{~kg} / \mathrm{m}^{2}$, presión arterial $115 / 70 \mathrm{mmHg}$, frecuencia cardiaca 80 I.p.m., $\mathrm{SpO}_{2} 89 \%$ a aire ambiente y ayuno $>8$ horas, oxígeno suplementario por puntas nasales a $6 \mathrm{l} / \mathrm{min}$. Se decide realizar sedación consciente para el procedimiento. Todos los fármacos infundidos fueron calculados según el peso ideal corregido. En el área preanestésica se inicia dexmedetomidina $0.5 \mu \mathrm{g} / \mathrm{kg} / \mathrm{h}$ en infusión continua por 25 minutos previo a iniciar el procedimiento. Durante todo momento el paciente es monitorizado de manera no invasiva (presión arterial, electrocardiograma, 
Tabla 1. Resultados de laboratorio del paciente

$\mathrm{TSH} 2.714 \mu \mathrm{UI} / \mathrm{ml}$

T3 total $1 \mathrm{ng} / \mathrm{ml}$

T3 libre $4.25 \mathrm{pg} / \mathrm{ml}$

T4 total $7.65 \mu \mathrm{g} / \mathrm{dl}$

T4 libre $1.13 \mathrm{ng} / \mathrm{ml}$

Ácido úrico 12.35 mg/dl

Hemoglobina glucosilada A1c 6.3\%

Insulina basal $15.8 \mathrm{mUI} / \mathrm{ml}$

Glucosa $116.4 \mathrm{mg} / \mathrm{dl}$

Sodio136 mmol//

Potasio $3.98 \mathrm{mmol} / \mathrm{l}$

Calcio $9.15 \mathrm{mg} / \mathrm{dl}$

Fósforo $2.78 \mathrm{mg} / \mathrm{dl}$

Cloro $96 \mathrm{mmol} / \mathrm{l}$

Urea $37 \mathrm{mg} / \mathrm{dl}$

BUN $17.06 \mathrm{mg} / \mathrm{dl}$

Creatinina $0.96 \mathrm{mg} / \mathrm{dl}$

Colesterol total $138.9 \mathrm{mg} / \mathrm{dl}$

Triglicéridos $140.2 \mathrm{mg} / \mathrm{dl}$

HDL $40.26 \mathrm{mg} / \mathrm{dl}$

LDL $92.25 \mathrm{mg} / \mathrm{dl}$

$\mathrm{DHL} 147.6 \mathrm{U} / \mathrm{I}$

FA $74 \mathrm{U} / \mathrm{l}$

GGT $29.5 \mathrm{U} / \mathrm{l}$

Proteínas totales $6.92 \mathrm{~g} / \mathrm{dl}$

Albúmina $3.76 \mathrm{~g} / \mathrm{dl}$

Hemoglobina $14.8 \mathrm{~g} / \mathrm{dl}$

Hematocrito $45.3 \%$

Plaquetas $381 \times 10^{3} / \mathrm{mm}^{3}$

Leucocitos $13.3 \times 10^{3} / \mathrm{mm}^{3}$

TP $14 \mathrm{~s}$

TTP $41.5 \mathrm{~s}$

INR 1.09

Gasometría venosa:

$\mathrm{pH} 7.46$

$\mathrm{pCO}_{2} 41 \mathrm{mmHg}$

$\mathrm{pO}_{2} 56 \mathrm{mmHg}$

$\mathrm{HCO}_{3} 29.2 \mathrm{mEq} / \mathrm{l}$

BE 4.9

$\mathrm{SO}_{2} 89.3 \%$

Lactato $1 \mathrm{mmol} / \mathrm{l}$

pulsioximetría), manteniendo Ramsay de 2. Se administra nebulización a base de lidocaína simple al $2 \%$, $100 \mathrm{mg}$ (Fig. 1), y al terminarla se administran dos disparos de espray bucofaríngeo de Lonol ${ }^{\circledR}$. Posteriormente se ingresa a la sala de endoscopia, donde se le administra sufentanilo $7.5 \mu \mathrm{g}$ en bolo en dosis única, se inicia propanidido a $25 \mathrm{mg} / \mathrm{kg} / \mathrm{h}$ y se continúa con dexmedetomidina a $0.5 \mu \mathrm{g} / \mathrm{kg} / \mathrm{h}$. A los 2 minutos de iniciada la infusión de propanidido, manteniendo

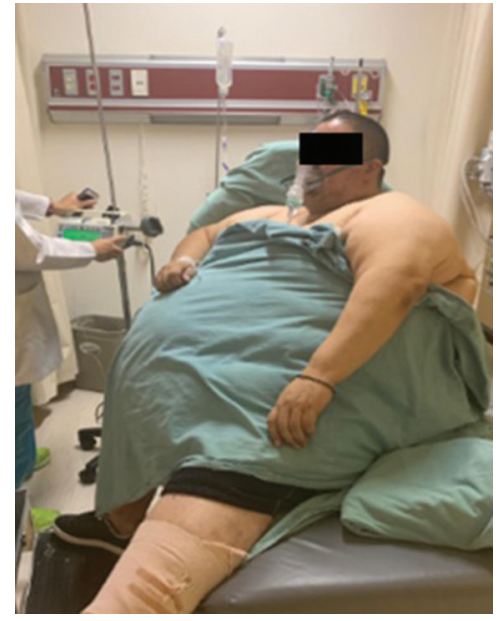

Figura 1. Inicio de la infusión de dexmedetomidina en el área preanestésica.

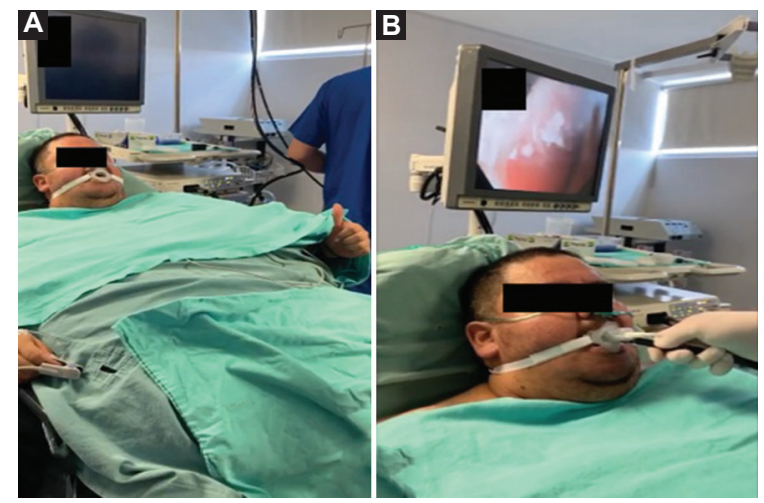

Figura 2. A: previo al inicio del procedimiento, paciente con Ramsay 3. B: inicio del procedimiento endoscópico, con el paciente tolerando la introducción del endoscopio sin presentar reflejo nauseoso ni molestia.

Ramsay de 3 (Fig. 2A), se inicia la colocación del balón intragástrico, tolerando el paciente adecuadamente la introducción del endoscopio sin presentar reflejo nauseoso ni molestia (Fig. 2B). Durante todo el procedimiento se mantuvo con Ramsay de 3-4. A 10 minutos de iniciado el procedimiento endoscópico se disminuye la tasa de infusión de propanidido a $20 \mathrm{mg} / \mathrm{kg} / \mathrm{h}$. Un minuto posterior a extraer el endoscopio el paciente presenta Ramsay de 2 con ENA 0, refiriendo no haber sentido ninguna molestia durante el evento, y se suspenden las infusiones. En ningún momento del procedimiento presentó desaturación ni apnea, $\mathrm{SpO}_{2} 99 \%$ con oxígeno suplementario, presión arterial $105 / 68 \mathrm{mmHg}$, frecuencia cardiaca 78 I.p.m. y frecuencia respiratoria 14 respiraciones por minuto (r.p.m.). Mantuvo la estabilidad hemodinámica durante 
el transanestésico (Fig. 3). La duración del procedimiento fue de 15 minutos en total y la duración de la anestesia de 30 minutos. Dosis total de dexmedetomidina $50 \mu \mathrm{g}$, dosis total de propanidido $640 \mathrm{mg}$ y dosis total de sufentanilo $7.5 \mu \mathrm{g}$. Balance de líquidos: ingresos $500 \mathrm{ml}(400 \mathrm{ml}$ de cristaloides y $100 \mathrm{ml}$ de paracetamol) y egresos $600 \mathrm{ml}$; balance $-100 \mathrm{ml}$. Medicamentos adyuvantes: omeprazol $40 \mathrm{mg}$, paracetamol $1 \mathrm{~g}$, hioscina $20 \mathrm{mg}$; todos por vía intravenosa. Sin reporte de incidentes durante la anestesia ni posterior a esta. Pasa a la sala de recuperación, manteniendo la estabilidad hemodinámica, consciente, Ramsay 2, con adecuada saturación con oxígeno suplementario. Tras 1 hora y 15 minutos de término de la anestesia, el paciente se encuentra sin dolor, hemodinámicamente estable, Aldrete 10 , presión arterial 115/68 mmHg, frecuencia cardiaca 80 I.p.m., frecuencia respiratoria 15 r.p.m. y $\mathrm{SpO}_{2} 89 \%$, y se le da de alta de la unidad de cuidados posanestésicos.

\section{Discusión}

Aún no contamos con el agente hipnótico ideal, pues actualmente ningún fármaco cumple con todas las características de esta definición; sin embargo, el propanidido presenta varias de ellas. Es un agente inductor no barbitúrico, cuyo principal efecto benéfico es su acción ultracorta, rápido inicio de acción y pronto despertar. Es un ácido derivado del feniloxiacético, aceite de clavo. Se presentaba con Cremophor $E L^{\circledR}$ como solubilizante, y fue suspendido debido a los graves efectos hemodinámicos causados por el solvente y no por el propanidido mismo $0^{10-12}$. Su mecanismo de acción se lleva a cabo al unirse a los receptores de tipo $A$ del ácido gamma-aminobutírico (GABA) e inhibir un sitio alostérico, actuando de manera agonista al GABA, realizando su función a nivel mesencefálico y bulbar ${ }^{10,13}$. La rapidez de la recuperación se debe a su conversión por la enzima plasmática pseudocolinesterasa y las esterasas hepáticas, mediante una rotura enzimática de la ligadura éster, a metabolitos ácidos carentes de actividad anestésica; la velocidad de degradación es directamente proporcional a la concentración del fármaco ${ }^{10,11}$. La dosis letal media es de $80 \mathrm{mg} / \mathrm{kg}^{10}$. Los reflejos pupilar, corneal y laríngeo permanecen activos, pero falta el reflejo faríngeo y hay relajación de los músculos maseteros en la mayor parte del periodo anestésico. Después de 10-12 minutos hay recuperación de la consciencia y el paciente puede abandonar el quirófano por su propio pie con una mínima somnolencia ${ }^{10}$.

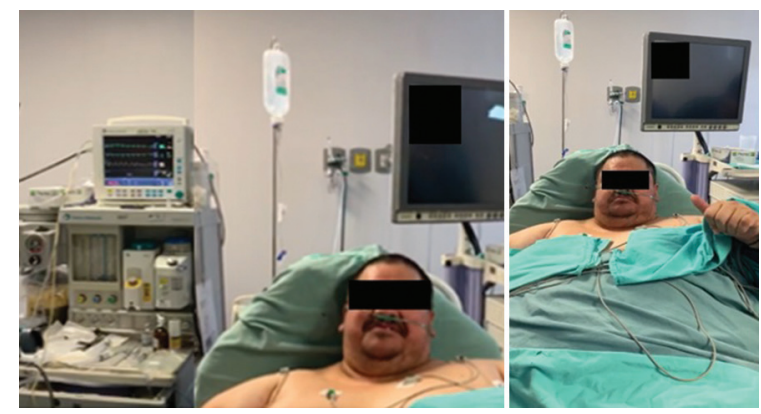

Figura 3. Posterior al término del evento endoscópico, el paciente presenta Ramsay 2 y ENA 0, manteniendo la estabilidad hemodinámica y una adecuada saturación por pulsioximetría.

Después de la inyección de $7 \mathrm{mg} / \mathrm{kg}$ aumenta el volumen respiratorio aproximadamente un $300 \%$ y la frecuencia respiratoria un $20 \%$. Esta hiperventilación persiste en promedio 40 segundos, se sigue de un periodo de hipoventilación o apnea que dura 2-5 segundos, y después vuelve a los niveles testigo ${ }^{11}$. Dosis menores tienen efectos menos intensos en la respiración y la saturación de oxígeno permanece a un nivel normal o un poco aumentada ${ }^{10}$. La hipotensión precede a la hiperventilación. Ambos efectos ocurren después de cada nueva inyección ${ }^{14,15}$. La presión arterial sistólica disminuye un 10\% y la diastólica un $15 \%$, en tanto que el pulso aumenta un $15 \%$ y luego vuelve a la normalidad en 2 minutos. Las posibles reacciones adversas incluyen temblores, movimientos involuntarios, taquicardia, tos, hipo, hipotensión, náusea, vómito y cefalea, en un porcentaje bajo ${ }^{10,11,16,17}$. Cuando contenía Cremophor $\mathrm{EL}^{\circledR}$ podían presentarse exantema o reacción alérgica e incluso efectos hemodinámicos adversos ${ }^{14}$. Está contraindicado en pacientes alérgicos a las especias, chile o clavo, y hay que tener precaución en los pacientes asmáticos 0 atópicos ${ }^{10}$. Además de las asignaciones para la edad (Tablas 2 y 3), las dosis necesitan ajuste según la robustez física ${ }^{16}$. Cualquier dosis producirá un efecto más largo en pacientes mayores de 40 años que en menores de 30 años $^{18}$. La nueva presentación es una ampolleta de $10 \mathrm{ml}$ que contiene $500 \mathrm{mg}$ de propanidido para diluir en solución glucosada al $5 \%$ a razón de $25 \mathrm{mg} / \mathrm{ml}$; algunos autores recomiendan no diluirlo ${ }^{10}$. En el año 2000 se logró estabilizar el propanidido en una base de microemulsiones Macrogol HS 15, con lo que mantiene las mismas propiedades benéficas sin efectos alérgicos y con baja liberación de histamina, lo cual reduce los efectos adversos ${ }^{10}$. Tiene un inicio de 
Tabla 2. Dosis de propanidido para inducción según la edad de los pacientes ${ }^{10,14}$.

- Adultos ASA 1-2: 5-7 mg/kg por vía intravenosa con una latencia de 30 segundos (6 mg/kg en mujeres y $7 \mathrm{mg} / \mathrm{kg}$ en hombres)

- Adultos jóvenes ASA 2-4: 3-5 mg/kg

- Pacientes seniles o debilitados, mayores de 50 años: 4-5 mg/kg

- Mayores de 70 años: 3-4 mg/kg

- Niños: 7-8 mg/kg e incluso hasta $10 \mathrm{mg} / \mathrm{kg}$ para la inducción

Tabla 3. Dosis de propanidido para mantenimiento10

- Dosis de impregnación: $10 \mathrm{mg} / \mathrm{kg}$ durante 3 minutos - Mantenimiento: $37 \mathrm{mg} / \mathrm{kg} / \mathrm{h}$

acción de 20 segundos ${ }^{19}$ y una vida media de acción de 7-10 minutos, con una recuperación aceptable en 20-25 minutos.

En un estudio comparativo de propanidido y propofol para endoscopias, se concluyó que es útil para procedimientos cortos ambulatorios ${ }^{10}$. En conclusión, el propanidido ha demostrado su seguridad, eficacia y versatilidad en procedimientos cortos, logrando un despertar más rápido que el propofol ${ }^{10,20}$. Hay poca literatura acerca de la endoscopia en personas con obesidad ${ }^{21}$, por lo que se requieren más estudios.

\section{Agradecimientos}

Agradecemos a la Clínica de Obesidad y Cirugía Bariátrica, y al Departamento de Endoscopia Gastrointestinal, del Hospital General Dr. Manuel Gea, por su colaboración.

\section{Responsabilidades éticas}

Protección de personas y animales. Los autores declaran que para esta investigación no se han realizado experimentos en seres humanos ni en animales.

Confidencialidad de los datos. Los autores declaran que han seguido los protocolos de su centro de trabajo sobre la publicación de datos de pacientes.
Derecho a la privacidad y consentimiento informado. Los autores han obtenido el consentimiento informado de los pacientes y/o sujetos referidos en el artículo. Este documento obra en poder del autor de correspondencia.

\section{Financiamiento}

Los autores declaran que no existen fuentes de financiamiento públicas o privadas en la realización del presente.

\section{Conflicto de intereses}

Los autores declaran que no hay conflicto de intereses.

\section{Bibliografía}

1. Carron M, Safaee Fakhr B, Leppariello G, Foletto M. Perioperative care of the obese patient. Br J Surg. 2020;107:e39-e55.

2. Catheline JM, Roussel J, Bénichou J. Surgical treatment of obesity. Reanimation. 2006;15:433-8.

3. Jirapinyo P, Thompson CC. Sedation challenges: obesity and sleep apnea. Gastrointest Endosc Clin North Am. 2016;26:527-37.

4. Qadeer MA. Risk factors for hypoxemia during ambulatory gastrointestinal endoscopy in ASA I-II patients. Dig Dis Sci. 2008;54:1035-40.

5. Vargo J. Endoscopic sedation in the bariatric patient: skating on thin ice? Dig Dis Sci. 2014;59:2023-4.

6. Wani S, Azar R, Hovis CE, Hovis RM, Cote GA, Hall M, et al. Obesity as a risk factor for sedation-related complications during propofol-mediated sedation for advanced endoscopic procedures. Gastrointest Endosc. 2011;74:1238-47.

7. McVay T, Fang JC, Taylor L, Au A, Williams W, Presson AP, et al. Safety analysis of bariatric patients undergoing outpatient upper endoscopy with non-anesthesia administered propofol sedation. Obes Surg. 2017;27:1501-7.

8. Schreine MA, Fennerty MB. Endoscopy in the obese patient. Gastroenterol Clin North Am. 2010;39:87-97.

9. Goudra BG, Singh PM, Penugonda LC, Speck RM, Sinha AC. Significantly reduced hypoxemic events in morbidly obese patients undergoing gastrointestinal endoscopy: predictors and practice effect. J Anaesthesiol Clin Pharmacol. 2014;30:71-7.

10. Barraza Espinoza V. Propanidido en México: mitos y realidades. Rev Mex Anestesiol. 2017;40:S79-S89.

11. Conway CM, Ellis DB. Propanidid. Br J Anaesth. 1970;42:249-54.

12. Wyant GM, Zoerb DL. Propanidid - a new non-barbiturate intravenous anaesthetic. Can Anaesth Soc J. 1965;12:569-86.

13. Wang S, Liu Q, Li X, Zhao X, Qiu L, Lin J. Possible binding sites and interactions of propanidid and AZD3043 within the $\gamma$-aminobutyric acid type A receptor (GABAAR). J Biomol Struct Dyn. 2017;36:3926-37.

14. Cenani A, Brosnan RJ, Knych HK. In vitro and in vivo GABAA receptor interaction of the propanidid metabolite 4-(2-[diethylamino]-2- oxoethoxy)-3-methoxy-benzeneacetic acid. Pharmacology. 2018;1:10-6.

15. Zindler M. Cardiovascular effects of propanidid. Acta Anaesth Scand. 1965;17:59-61.

16. Clark MM, Swerdlow M. The use of propanidid for minor surgery. Br J Anaesth. 1966;38:823-6.

17. Johns G. Cardiac arrest following induction with propanidid - a case report. Br J Anaesth. 1970;42:74-7.

18. Swerdlow M, Moore BA. A dose-duration trial with propanidid. $\mathrm{Br} \mathrm{J}$ Anaesth. 1967;39:573-7.

19. Cutile Quispe VM, Rojas Tintaya I. Anestésicos generales intravenosos. Revista de Actualización Clínica. 2012;27:1345-9.

20. Carron M, Freo U, Ori C. Occurrence and care of an obese patient during a serious adverse event with propofol for an endoscopy procedure. Obes Surg. 2011;21:410-1.

21. Vargo JJ. Procedural sedation and obesity: waters left uncharted. Gastrointest Endosc. 2009;70:980-4. 